Article

\title{
Synthesis of Di-(2-ethylhexyl) Phosphoric Acid (D2EHPA)-Tributyl Phosphate (TBP) Impregnated Resin and Application in Adsorption of Vanadium(IV)
}

\author{
Ruwei Zheng ${ }^{1}$, Shenxu Bao ${ }^{1,2, *}$, Yimin Zhang ${ }^{1,2,3}$ and Bo Chen ${ }^{1}$ \\ 1 School of Resources and Environmental Engineering, Wuhan University of Technology, \\ Wuhan 430070, China; zhruwei@126.com (R.Z.); zym126135@126.com (Y.Z.); \\ bochen2012@whut.edu.cn (B.C.) \\ 2 Hubei Key Laboratory of Mineral Resources Processing and Environment, Wuhan 430070, China \\ 3 Hubei Collaborative Innovation Center for High Efficient Utilization of Vanadium Resources, \\ Hubei Provincial Engineering Technology Research Center of High Efficient Cleaning Utilization for Shale \\ Vanadium Resource, Wuhan University of Science and Technology, Wuhan 430081, China \\ * Correspondence: sxbao@whut.edu.cn; Tel.: +86-27-8721-2127
}

Received: 4 March 2018; Accepted: 5 May 2018; Published: 10 May 2018

\begin{abstract}
In order to improve the adsorption capability of solvent-impregnated resins (SIRs) for vanadium(IV) (V(IV)), the dual extractant (D2EHPA (Di-(2-ethylhexyl) phosphoric acid) and TBP (Tributyl phosphate)) impregnated resins (D-TIRs) were prepared by impregnating Amberlite ${ }^{\mathrm{TM}}$ XAD-16HP macroporous resins with the mixed extractant that is composed by different molar ratios of D2EHPA to TBP. The effects of the ratio of D2EHPA to TBP on the performance of D-TIRs were investigated. The results show that the impregnation ratio of the D-TIRs decreases gradually with the increasing proportion of TBP in the mixed extractant. The sole-TBP impregnated resins (TIRs) have no adsorption capability for $\mathrm{V}(\mathrm{IV})$, indicating that the adsorption of $\mathrm{V}(\mathrm{IV})$ is attributed to D2EHPA. The adsorption capacity of D-TIRs for V(IV) attained the maximum when the ratio of D2EHPA to TBP is 7:3 at $\mathrm{pH} 1.8$, and it can be improved by increasing the extractants concentration during the impregnation process. Adsorption isotherm indicates that the addition of TBP can increase the adsorption capacity of D-TIRs for V(IV) from 24.65 to $29.75 \mathrm{mg} / \mathrm{g}$ after $16 \mathrm{~h}$ reaction. Adsorption kinetics verifies that the addition of TBP can largely accelerate the adsorption equilibrium of V(IV) onto the D-TIRs and V(IV). Electrospray ionization (ESI) mass spectra and Fourier transform infrared spectra (FT-IR) analysis indicates that the addition of TBP to D2EHPA can make some dimeric D2EHPA change to monomers by breaking the hydrogen bonds of D2EHPA-dimers, leading to the result that the pseudo-second order kinetic for the adsorption of V(IV) onto the D2EHPA impregnated resins (DIRs) converts to the pseudo-first order kinetic for that onto the D-TIRs. Also, D-TIRs have better separation capability of $\mathrm{V}$ (IV) from $\mathrm{Fe}(\mathrm{II})$ and $\mathrm{Al}(\mathrm{III})$ in the vanadium leaching solution than DIRs.
\end{abstract}

Keywords: solvent-impregnated resins; vanadium; adsorption; separation; kinetics

\section{Introduction}

Vanadium, which is a rare element with unique physical and chemical properties, is widely used in alloys, catalyst, vanadium redox battery, and aerospace [1,2]. Sulfuric acid leaching is applied extensively in vanadium extraction from the vanadium-bearing shale due to its high leaching efficiency and its low energy consumption [3,4]. However, many undesirable impurities, such as $\mathrm{Fe}$ and $\mathrm{Al}$, 
are dissolved in the acid leaching solution together with vanadium [5], rendering the separation and recovery of vanadium from the complex leaching solution as a necessary process for the production of $\mathrm{V}_{2} \mathrm{O}_{5}$ [6-8].

Recently, some researchers paid more attention to the separation and recovery of vanadium by using solvent-impregnated resins (SIRs), taking into account of their distinctive selectivity, easy operability and environmental friendliness [9]. Di-(2-ethylhexyl) phosphoric acid (D2EHPA) has been widely used as an excellent extractant for the recovery of vanadium because of its low price, high extraction efficiency, and the effective separation of vanadium(IV) (V(IV)) from impurity ions $[10,11]$, therefore it was used by many researchers to prepare SIRs for the separation of vanadium [12-14]. Liang et al. [12] studied the adsorption and separation of V(IV) from the vanadium leaching solution containing Fe(III) and Al(III) with D2EHPA impregnated resins (DIRs). It was found that the adsorption capacity of DIRs for $\mathrm{V}(\mathrm{IV})$ is only $14.43 \mathrm{mg} / \mathrm{g}$ after $18 \mathrm{~h}$ reaction and that the process is time-consumed. Tang et al. [14] also investigated the vanadium adsorption onto DIRs. The prepared DIRs have adsorption capacity of $19.25 \mathrm{mg} / \mathrm{g}$ for V(IV) at the reaction time of $12 \mathrm{~h}$. From the studies mentioned above, it can be seen that the sole-extractant impregnated resins generally need a long time to achieve adsorption equilibrium with $\mathrm{V}(\mathrm{IV})$ and they also present unsatisfactory separation capability for the aimed ions, which may limit their application in industry.

TBP (tributyl phosphate) is a widely used surfactant or interface modifier for extractants [15], which can improve the extraction efficiency and enhance phase separation in solvent extraction [16]. In the solvent extraction of vanadium, TBP is commonly used together with D2EHPA to improve the separation factor for vanadium and impurities [17-20]. Ma et al. [17] studied the separation of V(IV) and Fe(III) from vanadium-bearing acid leaching solution. They found that V(IV) and Fe(III) can be effectively separated from the solution by fractional extraction with D2EHPA and TBP. Li et al. [18] also recovered vanadium from acid leaching solution using D2EHPA-TBP, and they obtained high-purity $\mathrm{V}_{2} \mathrm{O}_{5}(99.61 \%)$. Cheraghi et al. [20] investigated the thermodynamics and mechanisms of vanadium extraction by a mixture of D2EHPA and TBP. Their results show that TBP in this solvent extraction system can decrease the $\mathrm{P}=\mathrm{O}$ vibration intensity of D2EHPA as a modifier. In summary, D2EHPA and TBP are often used together for the extraction of vanadium from the complex vanadium-bearing solutions. However, to the best of our knowledge, almost no study focuses on the application of the dual extractant (D2EHPA and TBP) impregnated resins (D-TIRs) in the adsorption and separation of vanadium [9]. The effect of the TBP in the D-TIRs on the adsorption and separation of V(IV) deserves investigation.

In this research, the dual extractant solvent with different proportions of D2EHPA to TBP were used to prepare D-TIRs. The effects of the molar ratio of D2EHPA to TBP on the impregnation ratio and adsorption characteristics of vanadium were investigated. The mechanism of vanadium adsorption onto the D-TIRs was also studied. Our study proposes a novel method to enhance the adsorption performance of SIRs for vanadium.

\section{Materials and Methods}

\subsection{Materials}

Macroporous resin, Amberlite ${ }^{\circledR}$ XAD-16HP, which was supplied by Shanghai Anland Co., Ltd., China, was used as the support for the preparation of D-TIRs. The XAD-16HP resin is fabricated with phenyl ethylene-divinyl benzene and is one non-polar polymer. The properties of XAD-16HP are listed in Table 1. First, the resin was soaked in ethanol for $12 \mathrm{~h}$ to remove the remained monomers and other types of impurities that are produced in the fabrication process, followed by washing with deionized water, and then dried at $60{ }^{\circ} \mathrm{C}$ in a vacuum oven for $12 \mathrm{~h}$ before use [12]. 
Table 1. Properties of XAD-16HP resin.

\begin{tabular}{cc}
\hline Parameters & Value \\
\hline Specific surface area & $800 \mathrm{~m}^{2} / \mathrm{g}$ \\
Pore volume & $1.82 \mathrm{~mL} / \mathrm{g}$ \\
Average pore diameter & $15 \mathrm{~nm}$ \\
Particle size & $0.425-0.850 \mathrm{~mm}$ \\
Matrix & phenyl ethylene-divinyl benzene \\
\hline
\end{tabular}

D2EHPA and TBP were purchased from Sinopharm Chemical Reagent Co., Ltd., Shanghai, China. The petroleum ether with a boiling point range of $60-90{ }^{\circ} \mathrm{C}$ (Sinopharm Chemical Reagent Co., Ltd., Shanghai, China) was used as diluent to dilute D2EHPA and TBP for preparing D-TIRs.

The acid leaching solution of vanadium-bearing shale generally needs reduction by sodium sulfite (Sinopharm Chemical Reagent Co., Ltd., Shanghai, China) before solvent extraction because D2EHPA has satisfying separation capability for V(IV) from Fe(II) [21]. Thus, the simulated vanadium leaching solution containing $1500 \mathrm{mg} / \mathrm{L} \mathrm{V(IV),} 3000 \mathrm{mg} / \mathrm{L} \mathrm{Fe(II),} \mathrm{and} 6000 \mathrm{mg} / \mathrm{L} \mathrm{Al(III),} \mathrm{which} \mathrm{refers} \mathrm{to} \mathrm{the}$ actual acid leaching solution [8], was prepared by dissolving vanadyl sulfate $\left(\mathrm{VOSO}_{4} \cdot \mathrm{xH}_{2} \mathrm{O}\right)$, ferrous sulfate $\left(\mathrm{FeSO}_{4} \cdot 7 \mathrm{H}_{2} \mathrm{O}\right)$, and aluminum sulfate hydrate $\left(\mathrm{Al}_{2}\left(\mathrm{SO}_{4}\right)_{3} \cdot 18 \mathrm{H}_{2} \mathrm{O}\right)$ in deionized water (Millpore Milli- ${ }^{\circledR}{ }^{\circledR}$ Burlington, MA, USA). The pure vanadium solution containing $1500 \mathrm{mg} / \mathrm{L} \mathrm{V(IV)}$ was prepared by only dissolving vanadyl sulfate in deionized water. The $\mathrm{pH}$ of the solutions was adjusted by sulfuric acid with A.R. grade. Vanadyl sulfate, which was ordered from Alfa Aesar (Tianjin, China) Chemical Co. Ltd., ferrous sulfate hydrate, and aluminum sulfate hydrate, which were obtained from Sinopharm Chemical Reagent Co., Ltd., were all C.P. grade. Other of the chemicals used in this study were of analytical grade.

The vanadium concentration was determined by ferrous ammonium sulfate titration using 2-(phenylamino)-benzoic acid as indicator and the concentrations of other ions in solution were analyzed by inductively coupled plasma-optical emission spectroscopy (ICP-OES) (Optima 4300DV, Perkin-Elmer, Waltham, MA, USA). The $\mathrm{pH}$ of solution was measured by $\mathrm{pHS}-3 \mathrm{C}$ digital $\mathrm{pH}$ meter (INESA Scientific Instrument Co., Ltd., Shanghai, China). The Electrospray ionization (ESI) mass spectra of D2EHPA and the mixture of D2EHPA and TBP (organic phase) were recorded on an ESI mass spectrometer (LCQadvantage, Thermo Finnigan, San Jose, CA, USA). Fourier transform infrared spectra (FT-IR) of the extractants (organic liquid) were recorded on a Nicolet 6700 spectrometer (Thermo Fisher Scientific Co., Waltham, MA, USA) at room temperature.

\subsection{Preparation of the D-TIRs}

D2EHPA and TBP were firstly mixed according to different molar ratios and were diluted in petroleum ether, then the diluted solutions were mixed with the treated XAD-16HP resins (as the liquid-solid ratio of $20: 1 \mathrm{~mL} \cdot \mathrm{g}^{-1}$ ) in a constant temperature bath oscillator at a stirring rate of $160 \mathrm{rpm}$. After shaking for $16 \mathrm{~h}$, the polymer beads were separated by filtration using a Buchner funnel and they were washed with deionized water. Finally, the impregnated resins were evaporated at $60^{\circ} \mathrm{C}$ in a vacuum oven for $12 \mathrm{~h}$ in order to remove the solvent [12]. The impregnation ratio of the D-TIRs, $\eta(\%)$, i.e., the ratio of the loaded extractants to the D-TIRs, was calculated by Equation (1):

$$
\eta=\frac{m_{2}-m_{1}}{m_{2}} \cdot 100 \%
$$

where $m_{2}$ is the weight of the dry D-TIRs (g) and $m_{1}$ is the weight of the treated dry resins (g). It was confirmed that the ratio of D2EHPA to TBP in D-TIRs is consistent with that in the solvent during the impregnation process by gas chromatography $(6890 \mathrm{~N}$, Agilent, Santa Clara, CA, USA). 


\subsection{Static Adsorption Experiments}

Except for the separation capability experiments, all of the adsorption experiments used the pure vanadium solution. First, $0.5 \mathrm{~g}$ dry D-TIRs were added to $25 \mathrm{~mL}$ pure vanadium or simulated vanadium leaching solutions in a conical flask, and then the mixture was shaken (160 rpm) at a bath oscillator at $25^{\circ} \mathrm{C}$ for different contacting time. Subsequently, the mixture was filtrated. The amount of $\mathrm{V}(\mathrm{IV})$ adsorbed onto the SIRs, i.e., the adsorption capacity $\left(Q\left(\mathrm{mg} \cdot \mathrm{g}^{-1}\right)\right)$, the distribution coefficient $(D)$, and separation factor $(\beta)$ were calculated by Equations (2)-(4), respectively.

$$
\begin{gathered}
Q_{e}=\frac{\left(C_{0}-C_{e}\right) V}{m} \\
D=\frac{\left(C_{0}-C_{e}\right) \cdot V / m}{C_{e}} \\
\beta_{A / B}=\frac{D_{A}}{D_{B}}
\end{gathered}
$$

where $V$ is the volume of $\mathrm{V}(\mathrm{IV})$ solutions $(\mathrm{L}), \mathrm{C}_{0}$ and $C_{e}$ are initial and equilibrium concentration of $\mathrm{V}(\mathrm{IV})$ in the solutions $\left(\mathrm{mg} \cdot \mathrm{L}^{-1}\right)$, respectively, and $m$ is the weight of the dry D-TIRs that were used in adsorption process (g). $D_{A}$ and $D_{B}$ is the distribution coefficient of $\mathrm{V}(\mathrm{IV})$ and impurity ion (Fe(II) or $\mathrm{Al}(\mathrm{III})$ ) between $\mathrm{D}$-TIRs and solution, respectively. $\beta_{A / B}$ represents the separation factor of ion $\mathrm{A}$ over B.

\section{Results and Discussion}

\subsection{Effect of the Ratio of D2EHPA to TBP on the Impregnation Ratio}

The total concentration of the mixed extractant (D2EHPA and TBP) in the solvent (petroleum ether) was kept as $1 \mathrm{~mol} / \mathrm{L}$ during the impregnated process, and the influence of the ratios of D2EHPA to TBP on the impregnation ratios is shown in Figure 1.

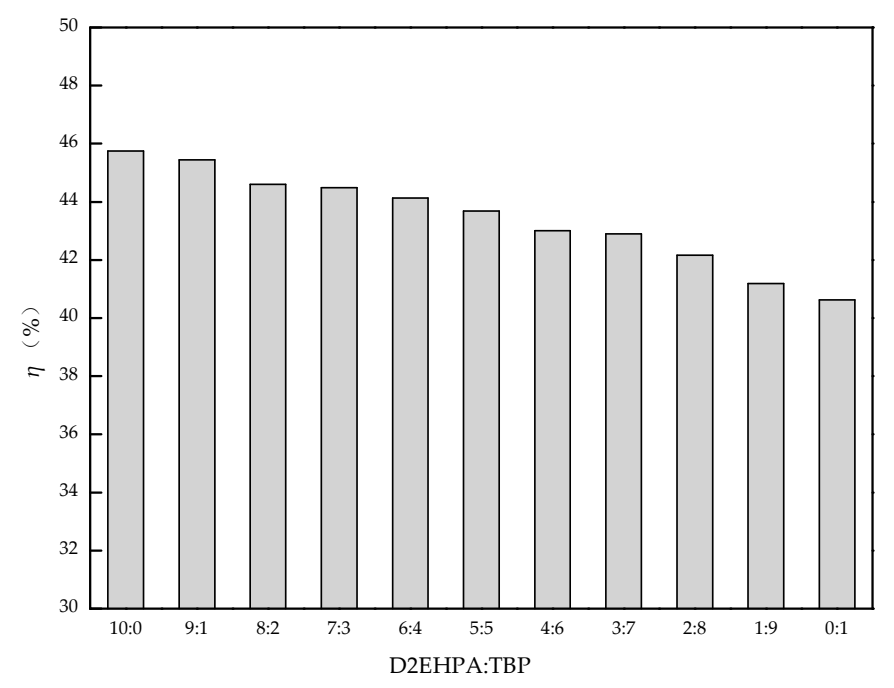

Figure 1. Effect of different ratios of D2EHPA to TBP on impregnation ratios.

It can be seen that the impregnation ratio of the D-TIRs decreases gradually with the decrease of the proportion of D2EHPA in the solvent (Figure 1). This may be caused by the different affinity of the support resin for D2EHPA and TBP. It is well known that D2EHPA is an acidic organophosphorus extractant and it is easy to form the non-polar dimer by hydrogen bond [21]. The non-polar dimer has higher affinity for the non-polar support resin than the polar TBP. Therefore, the impregnation ratio of the D-TIRs will decline with the increasing content of TBP in the mixed extractant. 


\subsection{Effect of Ratio of D2EHPA to TBP on Adsorption of V(IV)}

The adsorption capacity for V(IV) $\left(Q_{e}\right)$ was chosen as an indicator to evaluate the adsorption capability of the SIRs that were prepared in Section 3.1. The adsorption capacity of D-TIRs for V(IV) after $16 \mathrm{~h}$ reaction at different $\mathrm{pH}$ is shown in Figure 2.

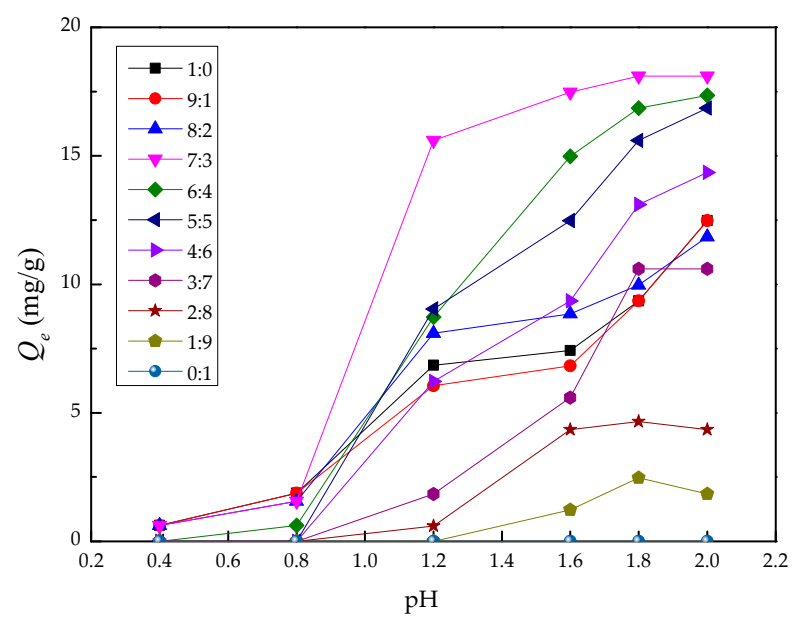

Figure 2. Adsorption capacity of different SIRs for $\mathrm{V}(\mathrm{IV})$ at different $\mathrm{pH}$ (concentration of the mixed extractant is $1 \mathrm{~mol} / \mathrm{L}$, adsorption time $16 \mathrm{~h}$ ).

As can be seen in Figure 2, the adsorption capacity of sole-TBP impregnated resins (TIRs) for $\mathrm{V}(\mathrm{IV})$ is zero, indicating that TBP cannot extract V(IV) in the resins, and V(IV) just is adsorbed by D2EHPA in the D-TIRs. Moreover, the D-TIRs with the molar ratio of D2EHPA to TBP $=7: 3$ exhibit an obviously higher adsorption capacity for V(IV) than other SIRs at wide $\mathrm{pH}$ range. The adsorption capacity of D-TIRs (D2EHPA:TBP = 7:3) for V(IV) increase with the increasing $\mathrm{pH}$. According to the previous research [21], the $\mathrm{P}-\mathrm{O}-\mathrm{H}$ bond in D2EHPA is responsible for the extraction of $\mathrm{VO}^{2+}$ through a cationic exchange mechanism, which is listed as follows:

$$
\mathrm{VO}^{2+}(a q)+2(\mathrm{HR})_{2(o)} \leftrightarrow \mathrm{VOR}_{2} \cdot 2 \mathrm{HR}_{(o)}+2 \mathrm{H}^{+}(a q)
$$

where $(\mathrm{HR})_{2}$ denotes the dimeric form of D2EHPA and the subscript $a q$ and $o$ indicate the aqueous phase and the organic phase, respectively.

It can be seen from Equation (5), that with the increase of $\mathrm{pH}$, the concentration of $\mathrm{H}^{+}$decreases and the reaction shifts towards the right, thus the adsorption capacity for $\mathrm{V}(\mathrm{IV})$ is increased. The adsorption capacity for V(IV) of the D-TIRs (D2EHPA:TBP $=7: 3$ ) can reach $18.1 \mathrm{mg} / \mathrm{g}$, while that of DIRs is only $9.35 \mathrm{mg} / \mathrm{g}$ at $\mathrm{pH} 1.8$, indicating that the addition of TBP in D-TIRs can significantly enhance the adsorption capacity for V(IV) after $16 \mathrm{~h}$ reaction. Thus, D2EHPA:TBP $=7: 3$ was selected as the best extractant ratio for preparing D-TIRs and $\mathrm{pH} 1.8$ was selected in the follow-up experiments.

\subsection{Effects of Extractant Concentration}

The concentration of dual extractant (D2EHPA:TBP $=7: 3$ was fixed) in the solvent was modified in the preparation of D-TIRs to investigate the effect of the extractant concentration on the impregnation ratio and the adsorption capacity for V(IV).

It is indeed possible to improve the resin's impregnation ratio and the adsorption capacity for $\mathrm{V}(\mathrm{IV})$ by increasing the concentration of the mixed extractant in the impregnation process (Figure 3). The impregnation ratio of the D-TIRs is approximately linear with the increasing extractant concentration, while the adsorption capacity for V(IV) of the D-TIRs firstly increase rapidly and then it grows slowly with the extractant's concentration. According to our previous research [14], 
D2EHPA is preferentially adsorbed in the micropores roughly as wall-spreading to form monolayer films, resulting in the relatively high utilization efficiency of the extractant at the low extractant concentration. However, with the increasing concentration of the mixed extractant, the extractant is more likely to accumulate in the macropores and/or mesopores as pore-filling, which leads to the higher impregnation ratio but a lower utilization efficiency. As a result, the increment of the adsorption capacity of the D-TIRs for vanadium slows down as the concentration of the extractant exceeds $1.2 \mathrm{~mol} / \mathrm{L}$ (Figure 3). It was found that the surface of the resin particles was covered with the mixed extractant when its concentration reached $2.0 \mathrm{~mol} / \mathrm{L}$. The extractant on the surface is easy to escape, which may affect the stability of the impregnated resins [22,23]. Thus, $1.8 \mathrm{~mol} / \mathrm{L}$ was adopted as the concentration of the mixed extractant for the impregnation process by considering the adsorption capacity and the stability, and the adsorption capacity of the D-TIRs for V(IV) can reach $28.76 \mathrm{mg} / \mathrm{g}$.

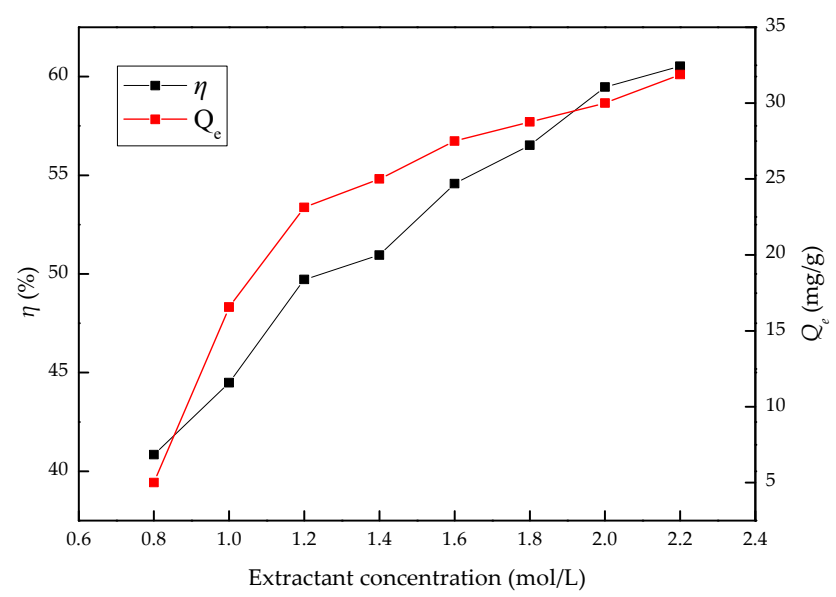

Figure 3. Effects of extractant concentration on the adsorption capacity and impregnation ratio of resins (D2EHPA:TBP = 7:3, adsorption time $16 \mathrm{~h}, \mathrm{pH} 1.8$ ).

\subsection{Adsorption Isotherm}

The adsorption isotherms were obtained to explore the effect of TBP addition on the adsorption capacity of D-TIRs (D2EHPA:TBP = 7:3 and the total concentration of D2EHPA and TBP is $1.8 \mathrm{~mol} / \mathrm{L}$ ) and DIRs (concentration of D2EHPA is $1.8 \mathrm{~mol} / \mathrm{L}$ ) for V(IV) after $16 \mathrm{~h}$ reaction, which are presented in Figure 4.

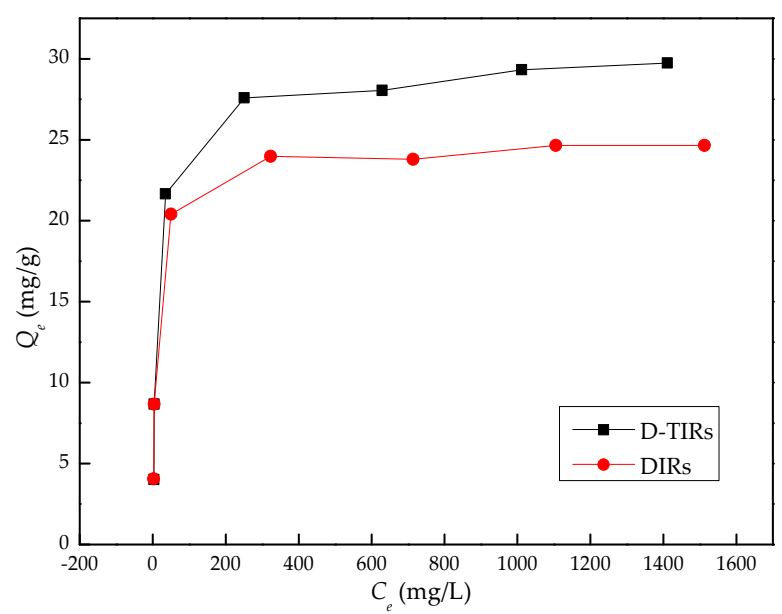

Figure 4. Loading capacity of SIRs for V(IV) at different vanadium concentrations. 
The loading capacity of SIRs (Figure 4) was determined using $0.5 \mathrm{~g}$ D-TIRs and DIRs, which were contacted with $25 \mathrm{~mL}$ vanadium(IV) solutions with different concentrations of V(IV). With the increase of the initial concentration of $\mathrm{V}(\mathrm{IV})$ in the solution, the adsorption capacity for V(IV) onto the two resins rapidly increases at the initial stage. When the equilibrium concentration of V(IV) in the solution achieves $300 \mathrm{mg} / \mathrm{L}$, the V(IV) loaded onto the D-TIRs and DIRs approximately reaches saturation. The loading capacity of D-TIRs and DIRs for V(IV) was found to be 24.65 and $29.75 \mathrm{mg} / \mathrm{g}$, respectively, verifying that $\mathrm{D}$-TIRs have higher adsorption capacity for $\mathrm{V}(\mathrm{IV})$ than DIRs after $16 \mathrm{~h}$ reaction.

Langmuir and Freundlich isotherms are commonly used to describe the adsorption of metals onto SIRs [24]. The Langmuir and Freundlich models are presented as Equations (6) and (7), respectively,

$$
\begin{gathered}
\frac{C_{e}}{Q_{e}}=\frac{1}{Q_{m} K_{L}}+\frac{C_{e}}{Q_{m}} \\
\log Q_{e}=\log K_{F}+\frac{1}{n} \log C_{e}
\end{gathered}
$$

where $Q_{e}$ is the amount of vanadium(IV) being adsorbed onto the SIRs at equilibrium(mg/g), $K_{L}$ is the Langmuir constant, and $Q_{m}$ is the maximum theoretical adsorption capacity of $\operatorname{SIRs}(\mathrm{mg} / \mathrm{g}) . K_{F}$ and $n$ are the Freundlich constants for adsorption capacity and adsorption intensity, respectively.

From the fitting parameters of Langmuir and Freundlich isotherms (Table 2), it can be concluded that Langmuir isotherm can better describe the adsorption process of V(IV) onto D-TIRs and DIRs as the coefficient of determination $\left(R^{2}\right)$ for the Langmuir isotherm is closer to 1 than that for the Freundlich isotherm. The maximum theoretical adsorption capacity $\left(Q_{m}\right)$ is $29.95 \mathrm{mg} / \mathrm{g}$ for D-TIRs and $24.80 \mathrm{mg} / \mathrm{g}$ for DIRs, indicating that the addition of TBP can increase the adsorption capacity of D-TIRs for V(IV) after $16 \mathrm{~h}$ reaction.

Table 2. Fitting parameters of Langmuir and Freundlich isotherm.

\begin{tabular}{ccccccc}
\hline \multirow{2}{*}{ SIRs } & \multicolumn{3}{c}{ Langmuir } & \multicolumn{3}{c}{ Freundlich } \\
\cline { 2 - 7 } & $\boldsymbol{Q}_{\boldsymbol{m}}$ & $\boldsymbol{K}_{\boldsymbol{L}}$ & $\boldsymbol{R}^{\mathbf{2}}$ & $\boldsymbol{n}$ & $\boldsymbol{K}_{\boldsymbol{F}}$ & $\boldsymbol{R}^{\mathbf{2}}$ \\
\hline D-TIRs & 29.95 & 0.0494 & 0.9995 & 3.5923 & 4.8301 & 0.7780 \\
DIRs & 24.80 & 0.0782 & 0.9998 & 4.0896 & 4.9962 & 0.7798 \\
\hline
\end{tabular}

\subsection{Adsorption Kinetics}

The adsorption capacity of the D-TIRs (D2EHPA:TBP $=7: 3$ and the total concentration of D2EHPA and TBP is $1.8 \mathrm{~mol} / \mathrm{L}$ ) and the DIRs (concentration of D2EHPA is $1.8 \mathrm{~mol} / \mathrm{L}$ ) for V(IV) at different times are compared in Figure 5.

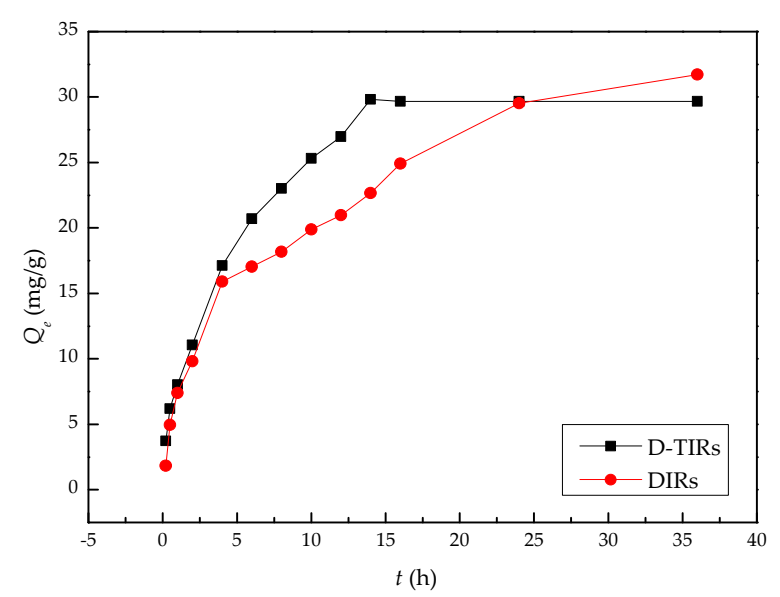

Figure 5. Absorption capacity of SIRs for V(IV) at different time. 
As can be seen from Figure 5, the adsorption capacity of two kinds of resins for V(IV) increases with the reaction time. The D-TIRs exhibit shorter equilibrium time than DIRs. The adsorption capacity of DIRs for V(IV) increases rapidly in the first $5 \mathrm{~h}$, and then slows down and still do not reach equilibrium till $36 \mathrm{~h}$. The adsorption of D-TIRs for V(IV) almost linearly increases with the increasing reaction time, and it reaches the equilibrium at about $14 \mathrm{~h}$. Because the amount of D2EHPA in D-TIRs is less than that in DIRs, so the saturation adsorption capacity for $\mathrm{V}(\mathrm{IV})$ of the former is slightly lower than that of the latter. However, it can be seen that the addition of TBP can significantly accelerate the adsorption rate of $\mathrm{V}(\mathrm{IV})$ onto D-TIRs and can reduce the adsorption equilibrium time.

Pseudo-first order kinetic equation (Equation (8)) and pseudo-second order kinetic equation (Equation (9)) are commonly used to depict the adsorption of metals onto SIRs [25].

$$
\begin{gathered}
\ln \left(Q_{e}-Q_{t}\right)=\ln Q_{e}-k_{1} t \\
\frac{t}{Q_{t}}=\frac{1}{k_{2} Q_{e}^{2}}+\frac{t}{Q_{e}}
\end{gathered}
$$

In these formulas, $Q_{t}$ is the adsorption capacity $(\mathrm{mg} / \mathrm{g})$ for $\mathrm{V}(\mathrm{IV})$ onto the resins at any moment, $t$ is the adsorption time $(\mathrm{h}), k_{1}\left(\mathrm{~h}^{-1}\right)$ and $k_{2}(\mathrm{~mol} /(\mathrm{L} \cdot \mathrm{h}))$ are the adsorption rate constant of pseudo-first order kinetic equation and pseudo-second kinetic equation, respectively. The linear fittings of the adsorption of V(IV) onto different impregnated resins are shown in Figure 6, and the fitting parameters are shown in Table 3.
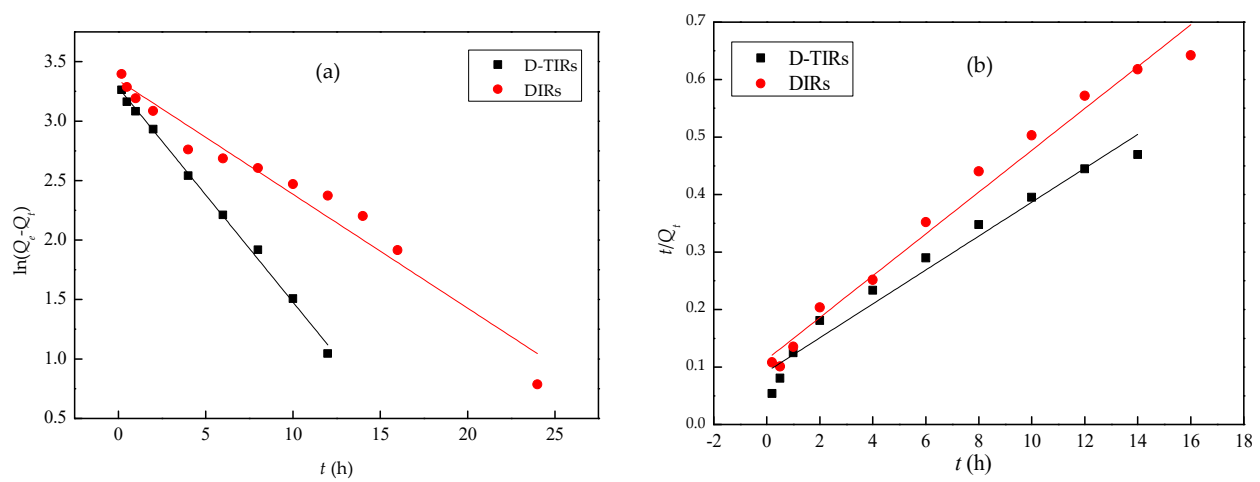

Figure 6. Fitting of the adsorption kinetics of V(IV) onto resins by (a) pseudo-first order model and (b) pseudo-second order model.

Table 3. Fitting parameters of the adsorption of V(IV) onto the SIRs.

\begin{tabular}{ccccccc}
\hline \multirow{2}{*}{ SIRs } & \multicolumn{3}{c}{ Pseudo-First Order } & \multicolumn{3}{c}{ Pseudo-Second Order } \\
\cline { 2 - 7 } & $\boldsymbol{R}^{\mathbf{2}}$ & $\boldsymbol{Q}_{\boldsymbol{e}} \mathbf{( \mathbf { m g } / \mathbf { g } )}$ & $\left.\boldsymbol{k}_{\mathbf{1}} \mathbf{( h}^{-\mathbf{1}}\right)$ & $\boldsymbol{R}^{\mathbf{2}}$ & $\boldsymbol{Q}_{\boldsymbol{e}} \mathbf{( \mathbf { m g } / \mathbf { g } )}$ & $\boldsymbol{k}_{\mathbf{2}} \mathbf{( \mathbf { m o l } / \mathbf { ( L } \cdot \mathbf { h } ) )}$ \\
\hline D-TIRs & 0.9967 & 26.65 & 0.1803 & 0.9657 & 33.90 & 0.0095 \\
DIRs & 0.9579 & 28.29 & 0.0957 & 0.9811 & 27.47 & 0.0117 \\
\hline
\end{tabular}

It can be seen from Figure 6 and Table 3 that the adsorption of V(IV) onto the D-TIRs conforms to the pseudo-first order model, while that onto the DIRs subjects to the pseudo-second order model. The reaction rate $k_{1}$ for D-TIRs is twice as much as that for DIRs, which verifies that TBP can accelerate the adsorption of V(IV) onto the SIRs [25].

The ESI mass spectra of D2EHPA and the mixture of D2EHPA and TBP, which are shown in Figure 7, are used to investigate the molecular forms of D2EHPA before and after the addition of TBP [26]. It can be seen that D2EHPA mainly exists as dimers (see the remarkable peak at $m / z=645.0$ in Figure 7a) [26]. However, it is obvious to see that two new peaks appear at $m / z=321.3$ and $m / z=531.1$, which are assigned to D2EHPA monomers and to TBP dimers, respectively, in the mixture 
of D2EHPA and TBP (Figure 7b). This result indicates that part hydrogen bonds linking D2EHPA monomers as dimers (Figure 8a) may be broken and some D2EHPA dimers are changed to monomeric D2EHPA (Figure 8b) after the addition of TBP. This also can be verified by the FT-IR spectra of different extractants (Figure 9).
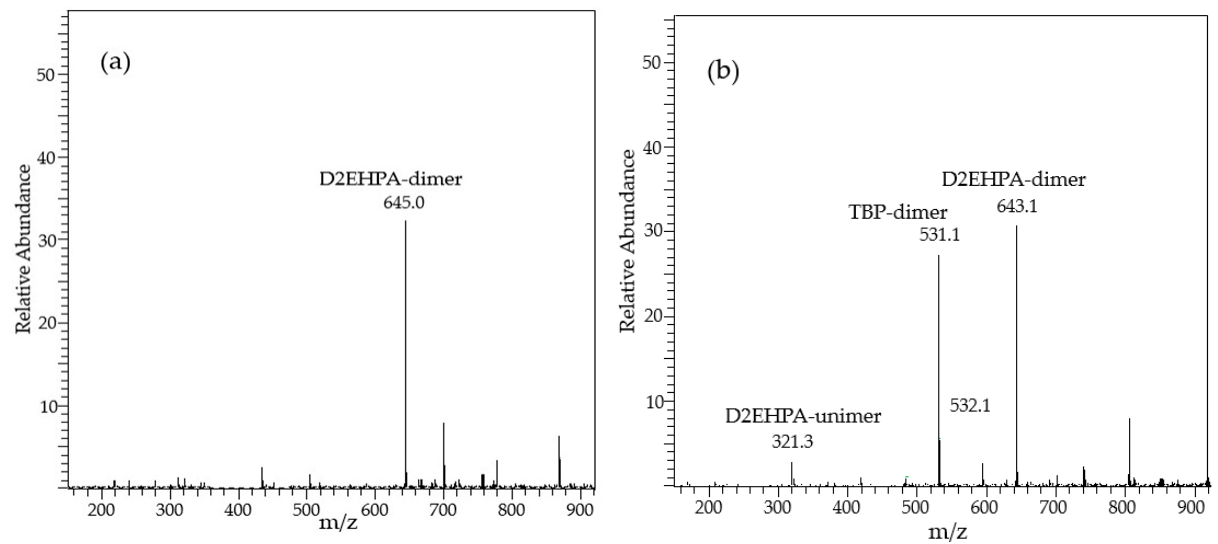

Figure 7. Electrospray Ionization (ESI) mass spectra of (a) D2EHPA and (b) mixture of D2EHPA and $\operatorname{TBP}(7: 3)$.

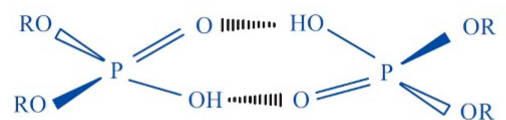

(a)

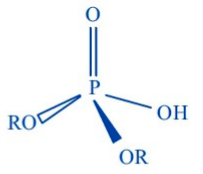

(b)

Figure 8. Proposed structure of (a) dimer of D2EHPA and (b) monomeric D2EHPA.

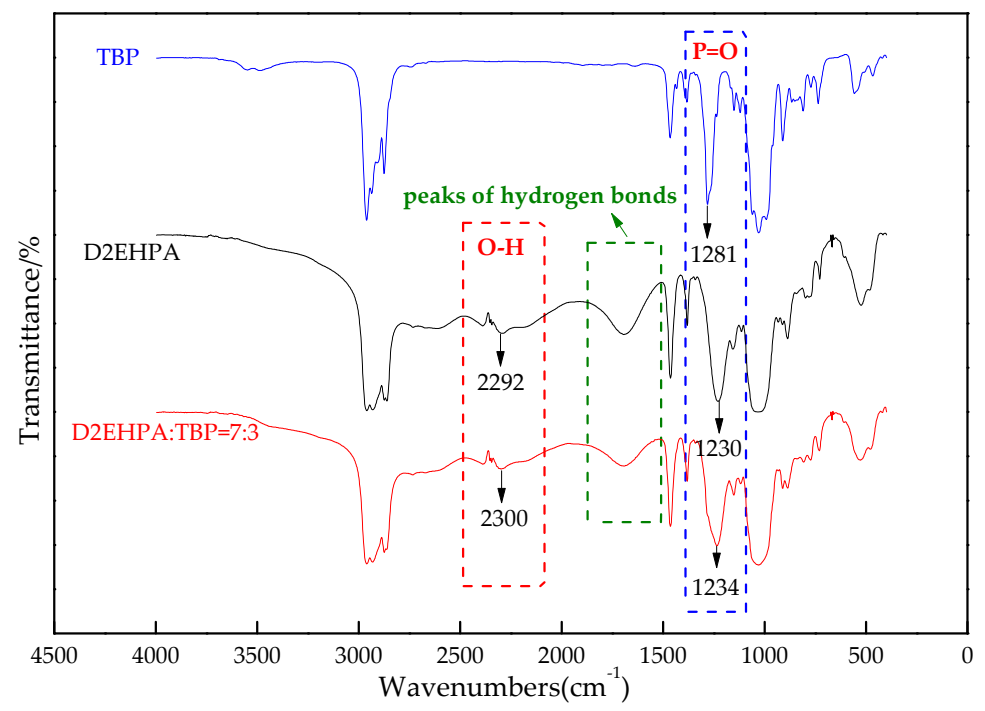

Figure 9. Fourier transform infrared spectra (FT-IR) spectra of TBP, D2EHPA, and mixture of D2EHPA to $\mathrm{TBP}=7: 3$.

In Figure 9, the peaks at 2292 and $2300 \mathrm{~cm}^{-1}$ are assigned to the $\mathrm{O}-\mathrm{H}$ vibration [21]. Three strong absorption peaks at 1230,1234 , and $1281 \mathrm{~cm}^{-1}$ were assigned to the $\mathrm{P}=\mathrm{O}$ vibration $[21,26]$. According to Griffiths's theory [27], the weakening of the hydrogen bonds will increase the electron cloud density and the chemical bond constant of the $\mathrm{O}-\mathrm{H}$ and $\mathrm{P}=\mathrm{O}$ bonds, leading the vibration absorption peaks to 
move to a higher wavenumber. It can be seen from Figure 9 that the vibration absorption peaks of $\mathrm{O}-\mathrm{H}$ and $\mathrm{P}=\mathrm{O}$ in D2EHPA shift to higher wavenumbers (2292 to $2300 \mathrm{~cm}^{-1}$ for $\mathrm{O}-\mathrm{H}$ and 1230 to $1234 \mathrm{~cm}^{-1}$ for $\mathrm{P}=\mathrm{O}$ ) after the addition of TBP, indicating that the amount of hydrogen bonds in D2EHPA may be reduced, which can also be verified by the weakening of hydrogen bonds intensity in the mixture when compared with those in D2EHPA. Thus, some dimeric D2EHPA are changed to the monomeric form, and the reaction between V(IV) and D2EHPA is also changed from Equation (5) to Equation (10):

$$
\mathrm{VO}^{2+}{ }_{(a q)}+2 \mathrm{HR}_{(o)} \leftrightarrow \mathrm{VOR}_{2(o)}+2 \mathrm{H}^{+}{ }_{(a q)}
$$

where HR denotes the monomeric D2EHPA.

By comparing Equation (5) with Equation (10), it is easy to see that the monomeric D2EHPA can provide more reaction sites than the D2EHPA dimers, and the reaction sites in the D-TIRs are more sufficient for V(IV) than those in the DIRs. Thus, the adsorption rate of V(IV) in the D-TIRs is faster than in the DIRs (Figure 5), and the adsorption process is also converted from pseudo-second order process for DIRs to pseudo-first order reaction for D-TIRs because the latter is commonly used to depict the reactions with sufficient reactants [25].

\subsection{Separation of $V(I V)$ from $\mathrm{Fe}(\mathrm{II})$ and $\mathrm{Al}(\mathrm{III})$}

The distribution coefficient of metals and the separation factors of V(IV) over metals onto the D-TIRs and the DIRs were shown in Figures 10 and 11, respectively.

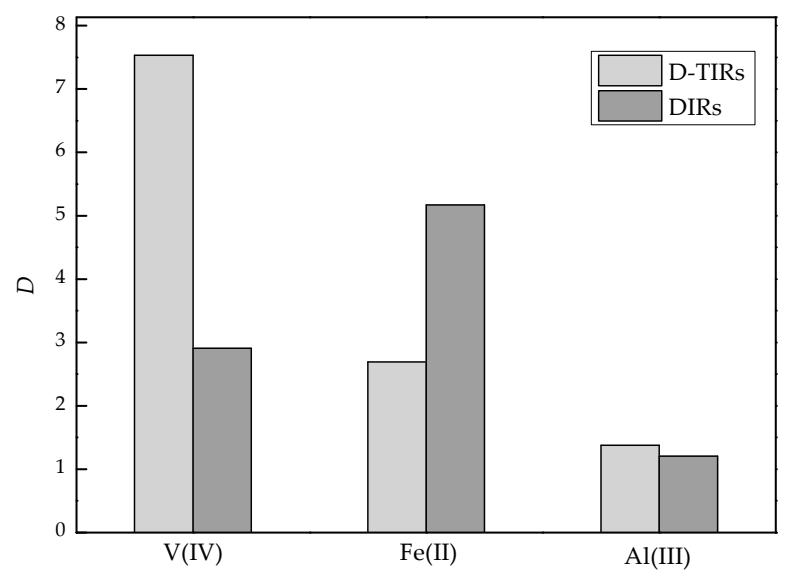

Figure 10. Distribution coefficient of $\mathrm{V}(\mathrm{IV}), \mathrm{Fe}(\mathrm{II})$, and $\mathrm{Al}(\mathrm{III})$ onto resins.

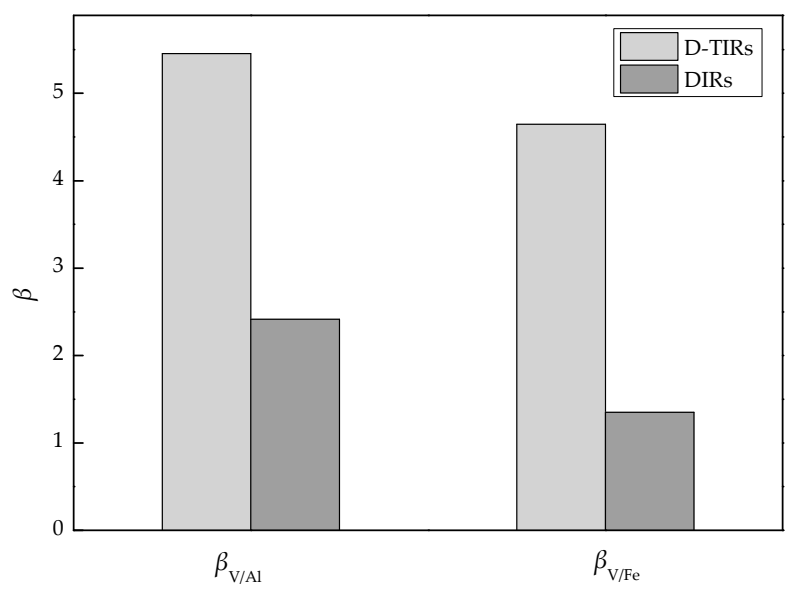

Figure 11. Separation factor of $\mathrm{V}(\mathrm{IV})$ over $\mathrm{Al}(\mathrm{III})$, and $\mathrm{V}(\mathrm{IV})$ over $\mathrm{Fe}(\mathrm{II})$ onto resins. 
Figure 10 presents that the distribution coefficient of V(IV) onto the D-TIRs is twice as high as that onto the DIRs, indicating that D-TIRs have much better adsorption capability for V(IV) than the DIRs. The distribution coefficient Fe(II) onto the D-TIRs is lower than that onto the DIRs, indicating that the D-TIRs is more better for the separation of V(IV) from Fe(II) than the DIRs. As can be seen from Figure 11, the separation factor $\beta_{\mathrm{V} / \mathrm{Al}}, \beta_{\mathrm{V} / \mathrm{Fe}}$ of D-TIRs are both higher than those of DIRs, which also verifies that the novel impregnated resins, D-TIRs, have a better capability for separating V(IV) from the acid leaching solution.

\section{Conclusions}

(1) The impregnation ratio of the D-TIRs gradually reduces with the increase of the proportion of TBP. The adsorption capacity of the D-TIRs for V(IV) can be significantly improved when the ratio of D2EHPA to TBP is 7:3 at $\mathrm{pH} 1.8$ after $16 \mathrm{~h}$ reaction.

(2) The adsorption capacity of the D-TIRs for V(IV) was improved by increasing the concentration of the mixed extractant during the impregnation process. The impregnation ratio and the adsorption capacity of D-TIRs increase with the increasing mixed extractant concentration. The adsorption capacity of D-TIRs for V(IV) can reach $28.76 \mathrm{mg} / \mathrm{g}$ when the mixed extractant concentration is $1.8 \mathrm{~mol} / \mathrm{L}$. Adsorption isotherm indicates that the addition of TBP can increase the theoretical adsorption capacity of D-TIRs for V(IV) from 24.80 to $29.95 \mathrm{mg} / \mathrm{g}$ after $16 \mathrm{~h}$ reaction.

(3) D-TIRs present a shorter equilibrium time and a higher adsorption capacity for V(IV) than DIRs in $24 \mathrm{~h}$. This is because that the addition of TBP to D2EHPA breaks some hydrogen bonds of D2EHPA-dimer, which increases the reaction sites of D2EHPA for V(IV) and leads to the conversion of the adsorption process from pseudo-second order process for DIRs to pseudo-first order reaction for D-TIRs

(4) The distribution coefficient of V(IV) onto the D-TIRs is higher than that onto the DIRs, and the separation factor $\beta_{\mathrm{V} / \mathrm{Al}}$ and $\beta_{\mathrm{V} / \mathrm{Fe}}$ for the D-TIRs are also obviously improved. This may be a potential method to improve the adsorption rate and the separation capability of the SIRs by preparing dual extractant impregnated resins that are used in metallurgy, chemical engineering, and environment fields.

Author Contributions: S.B. and R.Z. conceived and designed the experiments; R.Z. and B.C. performed the experiments; R.Z., S.B. and B.C. analyzed the data; S.B. and Y.Z. contributed reagents/materials/analysis tools; R.Z. and S.B. wrote the paper.

Acknowledgments: This research was supported by National Natural Science Foundation of China (51404177), National Key Science-Technology Support Programs of China (2015BAB03B05) and the Fundamental Research Funds for the Central Universities (WUT: 2017II34GX).

Conflicts of Interest: The authors declare no conflict of interest.

\section{References}

1. Zhang, Y.M.; Bao, S.X.; Liu, T.; Chen, T.J.; Huang, J. The technology of extracting vanadium from stone coal in China: History, current status and future prospects. Hydrometallurgy 2011, 109, 116-124. [CrossRef]

2. Zhang, G.; Zhang, Y.; Bao, S.; Huang, J.; Zhang, L. A novel eco-friendly vanadium precipitation method by hydrothermal hydrogen reduction technology. Minerals 2017, 7, 182. [CrossRef]

3. Chen, F.; Zhang, Y.; Huang, J.; Liu, T.; Xue, N. Mechanism of enhancing extraction of vanadium from stone coal by roasting with $\mathrm{MgO}$. Minerals 2017, 7, 33. [CrossRef]

4. Cai, Z.; Zhang, Y.; Liu, T.; Huang, J. Mechanisms of Vanadium recovery from stone coal by novel $\mathrm{BaCO}_{3} / \mathrm{CaO}$ composite additive roasting and acid leaching technology. Minerals 2016, 6, 26. [CrossRef]

5. Li, M.; Wei, C.; Qiu, S.; Zhou, X.; Li, C.; Deng, Z. Kinetics of vanadium dissolution from black shale in pressure acid leaching. Hydrometallurgy 2010, 104, 193-200. [CrossRef]

6. Yang, X.; Zhang, Y.; Bao, S. Preparation high purity $\mathrm{V}_{2} \mathrm{O}_{5}$ from a typical low-grade refractory stone coal using a pyro-hydrometallurgical process. Minerals 2016, 6, 69. [CrossRef] 
7. Li, W.; Zhang, Y.; Liu, T.; Huang, J.; Wang, Y. Comparison of ion exchange and solvent extraction in recovering vanadium from sulfuric acid leach solutions of stone coal. Hydrometallurgy 2013, 131-132, 1-7. [CrossRef]

8. Yang, X.; Zhang, Y.; Bao, S.; Shen, C. Separation and recovery of vanadium from a sulfuric-acid leaching solution of stone coal by solvent extraction using trialkylamine. Sep. Purif. Technol. 2016, 164, 49-55. [CrossRef]

9. Bao, S.; Tang, Y.; Zhang, Y.; Liang, L. Recovery and separation of metal ions from aqueous solutions by solvent-impregnated resins. Chem. Eng. Technol. 2016, 39, 1377-1392. [CrossRef]

10. Hu, G.; Chen, D.; Wang, L.; Liu, J.; Zhao, H.; Liu, Y.; Qi, T.; Zhang, C.; Yu, P. Extraction of vanadium from chloride solution with high concentration of iron by solvent extraction using D2EHP. Sep. Purif. Technol. 2014, 125, 59-65. [CrossRef]

11. Hughes, M.A.; Biswas, R.K. The kinetics of vanadium (IV) extraction in the acidic sulphate-D2EHPA-n-hexane system using the rotating diffusion cell technique. Hydrometallurgy 1991, 26, 281-297. [CrossRef]

12. Liang, L.; Bao, S.; Zhang, Y.; Tang, Y. Separation and recovery of V(IV) from sulfuric acid solutions containing $\mathrm{Fe}(\mathrm{III})$ and $\mathrm{Al}(\mathrm{III})$ using bis(2-ethylhexyl) phosphoric acid impregnated resin. Chem. Eng. Res. Des. 2016, 111, 109-116. [CrossRef]

13. Lee, G.S.; Uchikoshi, M.; Mimura, K.; Isshiki, M. Separation of major impurities Ce, Pr, Nd, Sm, Al, Ca, Fe, and Zn from La using bis(2-ethylhexyl) phosphoric acid (D2EHPA)-impregnated resin in a hydrochloric acid medium. Sep. Purif. Technol. 2010, 71, 186-191. [CrossRef]

14. Tang, Y.; Bao, S.; Zhang, Y.; Liang, L. Effect of support properties on preparation process and adsorption performances of solvent impregnated resins. React. Funct. Polym. 2017, 113, 50-57. [CrossRef]

15. Baaden, M.; Burgard, M.; Wipff, A.G. TBP at the water-oil interface: The effect of TBP concentration and water acidity investigated by molecular dynamics simulations. J. Phys. Chem. B 2001, 105, 11131-11141. [CrossRef]

16. Fatmehsari, D.H.; Darvishi, D.; Etemadi, S.; Hollagh, A.E.; Alamdari, E.K.; Salardini, A.A. Interaction between TBP and D2EHPA during $\mathrm{Zn}, \mathrm{Cd}, \mathrm{Mn}, \mathrm{Cu}, \mathrm{Co}$ and Ni solvent extraction: A thermodynamic and empirical approach. Hydrometallurgy 2009, 98, 143-147. [CrossRef]

17. Ma, Y.; Wang, X.; Wang, M.; Jiang, C.; Xiang, X.; Zhang, X. Separation of V(IV) and Fe(III) from the acid leach solution of stone coal by D2EHPA/TBP. Hydrometallurgy 2015, 153, 38-45. [CrossRef]

18. Li, X.; Deng, Z.; Wei, C.; Li, C.; Li, M.; Fan, G.; Huang, H. Solvent extraction of vanadium from a stone coal acidic leach solution using D2EHPA/TBP: Continuous testing. Hydrometallurgy 2015, 154, 40-46. [CrossRef]

19. Li, X.; Wei, C.; Deng, Z.; Li, M.; Li, C.; Fan, G. Selective solvent extraction of vanadium over iron from a stone coal/black shale acid leach solution by D2EHPA/TBP. Hydrometallurgy 2011, 105, 359-363. [CrossRef]

20. Cheraghi, A.; Ardakani, M.S.; Alamdari, E.K.; Fatmesari, D.H.; Darvishi, D.; Sadrnezhaad, S.K. Thermodynamics of vanadium (V) solvent extraction by mixture of D2EHPA and TBP. Int. J. Miner. Process. 2015, 138, 49-54. [CrossRef]

21. Shi, Q.; Zhang, Y.; Huang, J.; Liu, T.; Liu, H.; Wang, L. Synergistic solvent extraction of vanadium from leaching solution of stone coal using D2EHPA and PC88A. Sep. Purif. Technol. 2017, 181, 1-7. [CrossRef]

22. Trochimczuk, A.W.; Kabay, N.; Arda, M.; Streat, M. Stabilization of solvent impregnated resins (SIRs) by coating with water soluble polymers and chemical crosslinking. React. Funct. Polym. 2004, 59, 1-7. [CrossRef]

23. Kabay, N.; Cortina, J.L.; Trochimczuk, A.; Streat, M. Solvent-impregnated resins (SIRs)—Methods of preparation and their applications. React. Funct. Polym. 2010, 70, 484-496. [CrossRef]

24. Van Nguyen, N.; Lee, J.C.; Jha, M.K.; Yoo, K.; Jeong, J. Copper recovery from low concentration waste solution using Dowex G-26 resin. Hydrometallurgy 2009, 97, 237-242. [CrossRef]

25. Cortina, J.L.; Miralles, N. Kinetic studies on heavy metal ions removal by impregnated resins containing di-(2,4,4-trymethylpentyl) phosphinic acid. Solvent Extr. Ion Exch. 1997, 15, 1067-1083. [CrossRef]

26. Zhang, F.; Dai, J.; Wang, A.; Wu, W. Investigation of the synergistic extraction behavior between cerium (III) and two acidic organophosphorus extractants using FT-IR, NMR and mass spectrometry. Inorg. Chim. Acta 2017, 466, 333-342. [CrossRef]

27. Griffiths, P.R.; de Haseth, J.A. Fourier Transform Infrared Spectrometry; Wiley: New York, NY, USA, 1986; pp. 297-302.

(C) 2018 by the authors. Licensee MDPI, Basel, Switzerland. This article is an open access article distributed under the terms and conditions of the Creative Commons Attribution (CC BY) license (http:/ / creativecommons.org/licenses/by/4.0/). 